\title{
Poor long-term outcomes for cryptococcal meningitis in rural South Africa
}

\author{
Richard J Lessells, Portia C Mutevedzi, Tom Heller, Marie-Louise Newell
}

Objectives. To explore linkage to and retention in HIV care after an episode of cryptococcal meningitis (CM) in rural South Africa.

Design. A retrospective case series of adult individuals ( $\geq 16$ years old) with laboratory-confirmed CM from January - December 2007 at Hlabisa Hospital - a district hospital in northern KwaZuluNatal.

Outcome measures. Inpatient mortality and associated risk factors were analysed. The proportion alive and on antiretroviral therapy (ART) at 2 years was determined by linkage to the HIV treatment programme.

Results. One hundred and four individuals were identified with laboratory diagnosis of CM; 74/104 (71.2\%) with complete records were included in the analysis. Inpatient mortality was high (40.5\%) and was significantly associated with reduced conscious level (aHR $3.09,95 \%$ CI 1.30 - 7.33) and absence of headache (aHR 0.33 for headache, $95 \%$ CI $0.13-0.87)$. Only 8 individuals $(10.8 \%$ of all study subjects) were alive and receiving ART 2 years after the CM episode.

Conclusions. Long-term outcomes of $\mathrm{CM}$ are poor in routine practice. Interventions to strengthen linkage to HIV treatment and care and continuation of secondary fluconazole prophylaxis are critical.

S Afr Med J 2011;101:251-252.
Adult meningitis in areas of high HIV prevalence in southern Africa is most commonly caused by Cryptococcus neoformans. ${ }^{1}$ Short-term mortality in the antiretroviral therapy (ART) era varies widely, depending on treatment modalities and resources. ${ }^{2}$ Little is known about the longer-term prognosis in Africa, which would probably be an indicator of general effectiveness of the health system in managing complex HIV-associated conditions. Optimal management of cryptococcal meningitis (CM) involves induction therapy with amphotericin $\mathrm{B} \pm$ flucytosine, acute management of elevated cerebrospinal fluid (CSF) pressure, consolidation therapy with fluconazole, initiation of ART, and linkage to long-term outpatient care. ${ }^{2}$ We explored outcomes 2 years after an episode of cryptococcal meningitis in a rural area of South Africa.

\section{Methods}

Hlabisa Hospital is a 296-bed district hospital that, with 17 primary health care (PHC) clinics, serves 228000 people in northern KwaZuluNatal. The Hlabisa HIV Treatment \& Care Programme delivers ART at the hospital and all PHC clinics. ${ }^{3}$ This retrospective case series included all adult individuals ( $\geq 16 \mathrm{yrs}$ ) with a laboratory diagnosis of $\mathrm{CM}$, based on a positive cryptococcal antigen (CRAG) test \pm positive India ink examination from CSF, from 1 January - 31 December 2007. Hospital case records, pharmacy fluconazole records, and the HIV programme database were consulted regarding the acute hospital

Africa Centre for Health and Population Studies, University of KwaZulu-Natal, Mtubatuba, KwaZulu-Natal

Richard J Lessells, BSc, MB ChB, MRCP, DTM\&H, DipHIVMed

Portia C Mutevedzi, BSc, MSc

Marie-Louise Newell, MB, MSc, PhD

Hlabisa Hospital, Hlabisa, KwaZulu-Natal

Tom Heller, MD

UCL Institute of Child Health, London, UK

Marie-Louise Newell, MB, MSc, PhD episode and follow-up to 31 December 2009. Kaplan-Meier survival analysis and Cox regression were used to analyse inpatient mortality and associations with baseline clinical and laboratory features. To assess bias introduced by exclusion of individuals without hospital case records, characteristics of included and excluded patients were compared using the chi-squared (categorical variables) and Wilcoxon rank-sum tests (continuous variables). Ethical approval was received from the Hlabisa Hospital Ethics Committee.

\section{Results}

From 104 individuals, 140 positive CSF CRAG tests were identified; $74(71.2 \%)$ with complete hospital records were included. Median age was 35 years (IQR 30 - 44); 47.3\% were male; 47 (63.5\%) individuals were known HIV-infected at hospital admission, and 13 (17.6\%) were on ART. Median CD4 cell count $(N=45)$ was 49 cells/ $\mu$ l (IQR 16 - 86). The 74 patients most commonly presented with: headache (82.4\%), fever (37.8\%), vomiting $(35.1 \%)$, and reduced conscious level (33.8\%). Sixty-seven (90.5\%) of the 74 samples were positive on India ink examination; median CSF white blood cell (WBC) count was 20 cells/ml (IQR 4 - 56), and $22(29.7 \%)$ had a WBC count $<5 /$ ml. Only 26 patients (35.1\%) received amphotericin B, and only 6 received at least 14 days. Repeat therapeutic lumbar puncture was uncommon, documented in only 11 patients (14.9\%).

Inpatient mortality was $40.5 \%$ (30/74), all deaths occurring within 30 days of admission. The inpatient mortality rate was 2.99 per 100 person-days (95\% CI 2.09 - 4.28). Allowing for age, sex, CSF WBC count, amphotericin B treatment, CD4 cell count and ART, inpatient mortality was significantly associated with reduced conscious level at presentation (aHR 3.09, 95\% CI $1.30-7.33$ ) and with the presence of headache (aHR 0.33, 95\% CI 0.13 - 0.87).

Forty-four patients survived to hospital discharge. Thirty-five of these (35/44) (79.5\%) were confirmed HIV-infected by time of discharge ( 2 were counselled but refused testing; for 7 there was no documentation of counselling and testing). On discharge, all 44 were prescribed fluconazole, of whom $12(27.3 \%)$ also received ART ( 5 on ART at admission; 7 initiated ART during admission). Fig. 1 illustrates events following hospital discharge; 6 patients were re-admitted with cryptococcal meningitis, of whom 3 died in hospital. Only $9 / 44(20.5 \%)$ collected further fluconazole after the consolidation phase, and 9/32 (28.1\%) discharged on fluconazole 


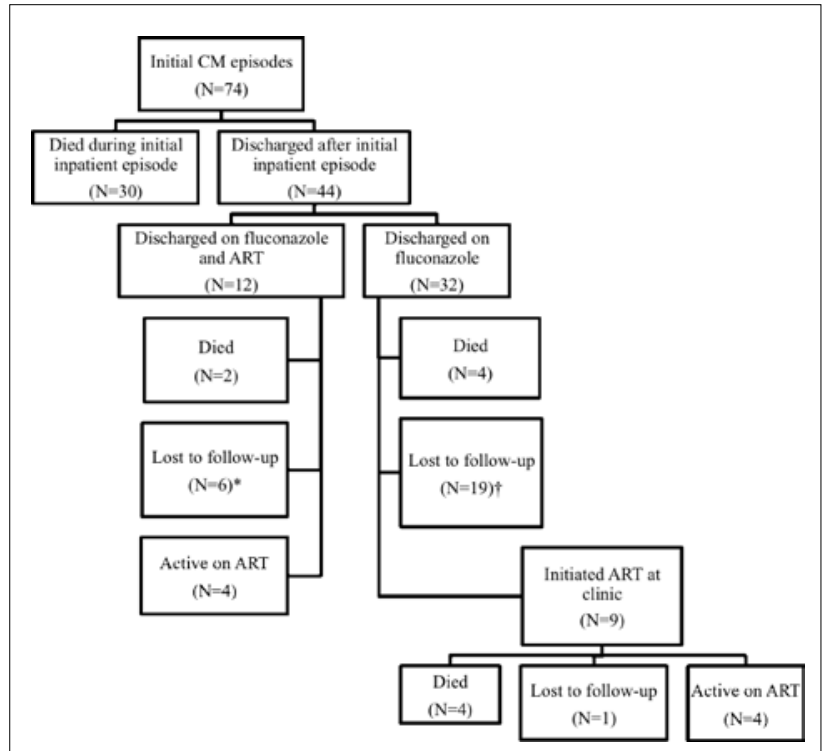

*Includes 5 individuals not seen after hospital discharge. $\dagger$ Includes 15 individuals not seen after hospital discharge.

Fig. 1. Study subject interventions and outcomes up to 31 December 2009 $(\mathrm{N}=74)$.

alone subsequently initiated ART. Only 8 (10.8\% of all subjects, $95 \%$ CI 8.7 - 12.8) were alive and receiving ART 2 years after the CM episode. For the 10 confirmed deaths post-discharge, the median time to death was 133 days (IQR 43 - 230).

There was no evidence that included patients $(N=74)$ differed from those excluded $(N=30)$ in terms of sex $(p=0.73)$, age $(p=0.13)$, CSF protein $(p=0.35)$, CSF glucose $(p=0.31)$ or CSF lymphocyte count $(p=0.26)$.

\section{Discussion}

These data from routine practice in a rural health district show poor long-term outcomes and highlight the challenges faced by overburdened health services in delivering comprehensive HIV care in high-prevalence settings. The short-term mortality is comparable with data from similar settings, ${ }^{4,5}$ but higher than reports from clinical trials or tertiary hospitals. ${ }^{6-10}$ About 1 in 10 of our patients were known to be alive on ART at 2 years, compared with: $60 \%$ at 12 months in a clinical trial in Cape Town; $70.9 \%$ at 12 months in a cohort in Uganda; $936.7 \%$ at 6 months in a Ugandan clinical trial; ${ }^{10}$ and $27 \%$ at 3 years in a trial in Zimbabwe. ${ }^{11}$ Inpatient mortality was associated with more severe disease (reduced conscious level and absence of headache), as also suggested in a neighbouring hospital in KwaZulu-Natal. ${ }^{5}$ High fungal burden is associated with mortality; our high rate of India ink positivity and reduced consciousness suggest advanced disease at presentation. ${ }^{12}$ Sub-optimal induction therapy and management of CSF pressure might have contributed to high inpatient mortality, although we could not confirm this. Amphotericin B treatment was uncommon, mainly owing to drug supply problems, in accordance with national-level data showing that more than half of $\mathrm{CM}$ cases receive sub-optimal induction treatment. ${ }^{13}$

Not all patients had their HIV status confirmed during admission, possibly owing to patient refusals, health care worker uncertainty about consent for confused or unconscious patients, or inadequate provision of counselling and testing services. The most striking finding was the high rate of loss to follow-up after hospital discharge; almost half were not seen again, which suggests poor linkage between hospital inpatient services and PHC clinics providing ongoing HIV care and treatment. Continuation of fluconazole after the consolidation phase was also uncommon, similar to other findings in South Africa. ${ }^{14,15}$ While some patients might have accessed fluconazole and ART elsewhere, the majority probably died.

There are limitations to this analysis, including those inherent in retrospective investigations. Retrieval of hospital records was incomplete, although we found no evidence that bias was introduced by excluding those without case records. Our numbers were too small to explore factors associated with long-term survival. Since this study, aspects of care have been improved: improved supply of amphotericin B, clear protocols for managing CSF pressure, enhanced HIV counselling and testing service for inpatients, and better linkage systems between hospital and PHC clinics. However, numerous challenges remain with the increasing numbers of CM cases in South Africa. ${ }^{16}$ Practical, implementable local care pathways, that encompass acute management and early post-discharge community support and enhanced clinical follow-up, must be developed and evaluated. By strengthening health systems, we can realise the full benefits from the scale-up of comprehensive HIV treatment and care.

We thank Ntombifuthi Mwelase and Nombuso Xaba for their assistance with data retrieval, and the staff of Hlabisa Hospital and the Hlabisa HIV Treatment and Care Programme. This work was supported by the Wellcome Trust (grant numbers 050534 and 075393). TH was supported by the Centre for International Migration and Development (CIM), and Gesellschaft für Technische Zusammenarbeit (GTZ), Federal Ministry of Economic Cooperation and Development, Germany.

\section{References}

Jarvis JN, Meintjes G, Williams A, Brown Y, Crede T, Harrison TS. Adult meningitis in a setting of high HIV and TB prevalence: findings from 4961 suspected cases. BMC Infect Dis 2010;10:67.

2. Sloan DJ, Dedicoat MJ, Lalloo DG. Treatment of cryptococcal meningitis in resource limited settings. Curr Opin Infect Dis 2009;22(5):455-463.

3. Houlihan CF, Bland RM, Mutevedzi PC, et al. Cohort profile: Hlabisa HIV Treatment and Care Programme. Int J Epidemiol 2010; $12 \mathrm{Feb}$. [Epub ahead of print].

4. Kisenge PR, Hawkins AT, Maro VP, et al. Low CD4 count plus coma predicts cryptococcal meningitis in Tanzania. BMC Infect Dis 2007;7:39.

5. Lightowler JV, Cooke GS, Mutevedzi P, Lessells RJ, Newell ML, Dedicoat M. Treatment of cryptococcal meningitis in KwaZulu-Natal, South Africa. PLoS One 2010;5(1):e8630.

6. Bicanic T, Meintjes G, Wood R, et al. Fungal burden, early fungicidal activity, and outcome in cryptococcal meningitis in antiretroviral-naive or antiretroviral-experienced patients treated with cryptococcal meningitis in antiretroviral-naive or antiretrovira
amphotericin B or fluconazole. Clin Infect Dis 2007;45(1):76-80.

amphotericin B or fluconazole. Clin Infect Dis 2007:45(1):76-80.
7. Bicanic T, Wood R, Meintjes G, et al. High-dose amphotericin B with flucytosine for the treatment of cryptococcal meningitis in HIV-infected patients: a randomized trial. Clin Infect Dis 2008;47(1):123130 .

8. Bisson GP, Nthobatsong R, Thakur R, et al. The use of HAART is associated with decreased risk of death during initial treatment of cryptococcal meningitis in adults in Botswana. J Acquir Immune Defic Syndr 2008;49(2):227-229.

. Kambugu A, Meya DB, Rhein J, et al. Outcomes of cryptococcal meningitis in Uganda before and after the availability of highly active antiretroviral therapy. Clin Infect Dis 2008;46(11):1694-1701.

. Longley N, Muzoora C, Taseera K, et al. Dose response effect of high-dose fluconazole for HIVassociated cryptococcal meningitis in southwestern Uganda. Clin Infect Dis 2008;47(12):1556-1561.

1. Makadzange AT, Ndhlovu CE. Takarinda $K$, et al. Early versus delayed initiation of antiretroviral

Makadzange AT, Ndhlovu CE, Takarinda K, et al. Early versus delayed initiation of antiretroviral therapy for concurrent HIV infection and cryptococcal meningitis in sub-saharan Africa. Clin Infect
Dis 2010;50(11):1532-1538.

2. Bicanic T, Muzoora C,

Bicanic 1, Muzoora C, Brouwer AE, et al. Independent association between rate of clearance of infection and clinical outcome of HIV-associated cryptococcal meningitis: analysis of a combined cohort of 262 patients. Clin Infect Dis 2009;49(5):702-709.

13. Govender N, Cohen C, Meiring S, et al. Trends in treatment of adults with incident cryptococcosis, South Africa, 2005-2008. San Fransisco, California: 17th Conference on Retroviruses and Opportunistic Infections, 2010

14. Collett $\mathrm{G}$, Parrish A. Fluconazole donation and outcomes assessment in cryptococcal meningitis. S Afr Med J 2007;97(3):175-176.

15. Jarvis JN, Meintjes G, Williams Z, Rebe K, Harrison TS. Symptomatic relapse of HIV-associated cryptococcal meningitis in South Africa: the role of inadequate secondary prophylaxis. S Afr Med J 2010;100(6):378-382.

16. Jarvis JN, Boulle A, Loyse A, et al. High ongoing burden of cryptococcal disease in Africa despite antiretroviral roll out. AIDS 2009;23(9):1182-1183. 